\title{
Correction to: Thermal biology of Tuta absoluta: demographic parameters and facultative diapause
}

\author{
Mateus Ribeiro de Campos ${ }^{1}$ (1) $\cdot$ Philippe Béarez ${ }^{1}$ Edwige Amiens-Desneux ${ }^{1} \cdot$ Luigi Ponti $^{2,3}$ (i) . \\ Andrew Paul Gutierrez ${ }^{3,4}$ (C) Antonio Biondi ${ }^{5}$ (i) $\cdot$ Abhijin Adiga $^{6}$ (D) Nicolas Desneux ${ }^{1}$
}

Published online: 25 November 2020

(c) Springer-Verlag GmbH Germany, part of Springer Nature 2020

\section{Correction to: Journal of Pest Science https://doi.org/10.1007/s10340-020-01286-8}

Formula, Equation 2 and the table 3 are published incorrectly in the original publication of the article. The correct version of the article is given below (Eq. 2, Table 3)

(i) The mean generation time formula should be read as:

$$
\tau=\operatorname{Ln} R_{0} / r_{m}
$$

(ii) Equation 2 should be read as:

$$
\text { with } T_{\text {mid }}=\frac{\left(T_{\max }-T_{\min }\right)}{2}
$$

The original article can be found online at https://doi.org/10.1007/ s10340-020-01286-8

Mateus Ribeiro de Campos

mateusrcampos@gmail.com

Antonio Biondi

antonio.biondi@unict.it

Nicolas Desneux

nicolas.desneux@univ-cotedazur.fr

1 INRAE, CNRS, UMR ISA, University Côte d'Azur, 06000 Nice, France

2 Agenzia nazionale per le nuove tecnologie, l'energia e lo sviluppo economico sostenibile (ENEA), Centro Ricerche Casaccia, Via Anguillarese 301, 00123 Roma, Italy

3 Center for the Analysis of Sustainable Agricultural Systems Global (CASAS Global), Kensington, CA 94707-1035, USA

4 College of Natural Resources, University of California, Berkeley, CA 94720, USA

5 Department of Agriculture, Food and Environment, University of Catania, Via Santa Sofia 100, Catania, Italy

6 University of Virginia, Charlottesville, VA 22904, USA 
(iii)

Table 3 Means $\left( \pm\right.$ SE) of net reproductive rate $\left(R_{0}\right)$, finite rate of increase $(\lambda)$, intrinsic rate of increase $\left(r_{m}\right)$ and mean generation time $(\tau)$ of Tuta absoluta under six constant temperatures estimated by bootstrap sampling (10,000 replicates)

\begin{tabular}{lllrl}
\hline $\begin{array}{l}\text { Temperatures } \\
\left({ }^{\circ} \mathrm{C}\right)\end{array}$ & \multicolumn{4}{l}{ Life table parameters of Tuta absoluta } \\
\cline { 2 - 5 } & $R_{0}$ (Offspring/Individual) & $\lambda\left(\right.$ days $\left.^{-1}\right)$ & $r_{\mathrm{m}}$ (days $\left.{ }^{-1}\right)$ & $\tau$ (days) \\
\hline 10 & $14.10 \pm 4.12 \mathrm{bc}$ & $1.02 \pm 3.38 \times 10^{-3} \mathrm{e}$ & $2.56 \times 10^{-2} \pm 3.30 \times 10^{-3} \mathrm{e}$ & $103.04 \pm 1.77 \mathrm{a}$ \\
15 & $25.17 \pm 5.56 \mathrm{ab}$ & $1.05 \pm 4.32 \times 10^{-3} \mathrm{~d}$ & $5.33 \times 10^{-2} \pm 4.10 \times 10^{-3} \mathrm{~d}$ & $60.57 \pm 1.27 \mathrm{~b}$ \\
20 & $32.72 \pm 6.49 \mathrm{a}$ & $1.08 \pm 4.96 \times 10^{-3} \mathrm{c}$ & $8.16 \times 10^{-2} \pm 4.58 \times 10^{-3} \mathrm{c}$ & $42.72 \pm 1.11 \mathrm{c}$ \\
25 & $36.53 \pm 9.64 \mathrm{a}$ & $1.15 \pm 1.34 \times 10^{-2} \mathrm{a}$ & $14.32 \times 10^{-2} \pm 1.17 \times 10^{-2} \mathrm{a}$ & $25.12 \pm 0.60 \mathrm{~cd}$ \\
30 & $11.33 \pm 2.74 \mathrm{c}$ & $1.10 \pm 1.24 \times 10^{-2} \mathrm{~b}$ & $9.79 \times 10^{-2} \pm 1.12 \times 10^{-2} \mathrm{~b}$ & $24.80 \pm 0.90 \mathrm{~cd}$ \\
33 & $3.13 \pm 1.50 \mathrm{~d}$ & $1.05 \pm 2.79 \times 10^{-2} \mathrm{cde}$ & $5.21 \times 10^{-2} \pm 2.69 \times 10^{-2} \mathrm{cde}$ & $21.91 \pm 1.89 \mathrm{~d}$ \\
\hline
\end{tabular}

Means followed by different letters in columns indicate significant differences among treatments (paired bootstrap test, $P<0.05$ )

Publisher's Note Springer Nature remains neutral with regard to jurisdictional claims in published maps and institutional affiliations. 